\title{
Usefulness of Non-Invasive Fetal RHD Genotyping towards Immunoprophylaxis Optimization
}

\author{
Sebastián Blanco ${ }^{a}$ b $\quad$ Virginia Soledad Giacomic ${ }^{c}$ Luciano Gabriel Slobodianiuk ${ }^{c}$ \\ María Celia Frutos $^{b}$ Luis Horacio Carrizo ${ }^{a, c}$ Gabriela Elvira Fanin ${ }^{c}$ Jorge Mario Culasso ${ }^{c}$ \\ Sandra Verónica Gallego a, b \\ ${ }^{a}$ Fundación Banco Central de Sangre, Córdoba, Argentina; \\ b Instituto de Virología Dr J.M.Vanella, Facultad de Ciencias Médicas, Universidad Nacional de Córdoba, Córdoba, Argentina; \\ c Servicio de Hemoterapia e Inmunohematologia, Hospital Materno-Provincial Dr. Raúl Felipe Lucini, Córdoba, Argentina
}

\section{Keywords}

Non-invasive prenatal diagnosis - Molecular blood group typing · Fetal RhD genotyping - Antenatal immunoprophylaxis - Hemolytic disease of the newborn

\section{Summary}

Introduction: Since anti-D immunoprophylaxis given to D-negative pregnant women is a blood product, blood donations have an impact on the availability of prophylactic doses. The Pan American Health Organization reported, in June 2017, that less than half of blood donors are volunteers in Latin America and the Caribbean. In these countries, guidelines for use of anti-D prophylaxis are still controversial. The aim of this study was to demonstrate the convenience of a simple and cost-effectivene non-invasive prenatal diagnostic assay for anti-D prophylaxis optimization in multiethnic populations. Methods: Cell-free fetal DNA from plasma samples of Dnegative pregnant women were analyzed by real-time PCR for simultaneous amplification of sequences of exons 5 and 10 of the RHD gene. Fetal RHD genotype was determined in 111 pregnant women. Neonates' phenotype was determined $72 \mathrm{~h}$ after birth. Results: Genotyping predicted fetal phenotype with 100\% accuracy. Prenatal diagnosis showed 78\% RHD-positive and $22 \%$ RHD-negative neonates. Conclusion: We demonstrated that, beyond the large genetic variation of the Rh system and the numerous $D$ variants present in multiethnic groups, non-invasive fetal $R H D$ genotyping using two sequences of the gene can be enough for clinical application in an admixed population. This robust technique of simple implementation allows to determine fetal RHD in maternal blood with high sensitivity, specificity, and accuracy. The introduction of fetal $R h D$ genotyping as part of an antenatal screening program constitutes a reliable manner to optimize anti-D prophylaxis; however, it has not been implemented so far in most American countries.

(c) 2018 S. Karger GmbH, Freiburg

\section{Introduction}

The introduction of postnatal prophylactic treatment with $\mathrm{RH}$ immunoglobulin (IgRH) in the 1960s allowed to reduce the prevalence of hemolytic diseases in fetuses and newborns (HDFNs) due to incompatibility in the RH system from 16 to $2 \%[1,2]$. The implementation of antenatal prophylaxis at weeks 28-34 of gestation in the 1970s, in addition to postnatal prophylaxis, reduced such values to $0.1-0.3 \%[3,4]$.

Anti-D immunoglobulin is a limited biological product that cannot be manufactured; it is obtained from apheresis donations from voluntary donors who have high titers of anti-D antibodies. This situation has an impact on the availability of IgRH doses, which are frequently scarce. In many countries, it is necessary to import IgRH because it is not produced locally and in other countries such as Argentina, still having blood product companies, not only the product but also the raw material have to be imported for fractionation due to shortage of donors and the lack of implementation of national programs for obtaining plasma with high anti-D titers $[5,6]$. For this reason, guidelines for the use of IgRH prophylaxis are still a controversial issue in some countries, especially if volunteer blood donors are not enough to guarantee sufficient and safe blood supplies to manufacture blood products. Since this is a

\section{KARGER}

(c) 2018 S. Karger GmbH, Freiburg

Fax +497614520714 
current matter in Latin America and the Caribbean, there is no uniformity of criteria regarding the indication of prophylaxis and management of D-negative pregnant women. In Argentina, some local public health authorities do not adhere to the national recommendations that suggest to administer prenatal prophylaxis between weeks 28 and 32 of gestation and only provide IgRH to Dnegative pregnant women at risk of fetal-obstetric hemorrhage and to D-negative women after the birth of a D-positive child.

Another issue that should be taken into consideration in terms of the rational use of IgRH is that since it is a biological product prepared from pooled plasma of immunized donors, it involves all the risks associated with human blood products, including transmission of different infections. There are several antecedents in this respect. Kenny-Walsh et al. [7] found batches of IgRH used in Ireland between 1977 and 1978 that were contaminated with hepatitis $\mathrm{C}$ virus (HCV) and detected this virus in a group of women that had received IgRH. Smith et al. [8] investigated the infectivity for HCV of IgRH batches prepared in Ireland between 1991 and 1994 and detected the virus in 44 women who had received IgRH. In addition, in Germany, hepatitis $G$ virus (GBV-C) sequences were detected in batches of anti-D immunoglobulin contaminated with $\mathrm{HCV}$, responsible for a HCV outbreak in 1979 [9]. Even though technology has evolved significantly in recent decades, and plasma manufacturing plants have pursued strategies to reduce the risk of transfusion-transmitted infections, a residual risk still persist, and emerging diseases are a constant challenge in transfusion practice.

Since the molecular basis of the gene encoding D antigen was recognized, techniques for prenatal $R H D$ genotyping have been applied for the management of D-negative pregnant women previously sensitized or at risk of immunization. The accurate prediction of fetal D status allows to administrate prenatal prophylaxis to D-negative women with D-positive fetuses and avoids unnecessary administration in cases of $\mathrm{D}$-negative fetuses, optimizing the available resources. Thus, the assessment of the risk of HDFN by determining the RHD genotype from the cell-free fetal DNA (cffDNA) in maternal blood implicates an improvement in IgRH prophylaxis, only comparable to that of the introduction of the prophylaxis itself at that time. Therefore, non-invasive genotyping of fetal $R H D$ status by analyzing cffDNA in maternal plasma has already been incorporated into routine clinical practice of many countries, causing a great impact on management protocols of $\mathrm{D}$-negative pregnant women [10]. In Belgium, since 2002, fetal RHD genotyping has been used during the follow-up of D-negative pregnant women for an accurate indication of prophylaxis, and, in parallel, prevention policies have been implemented, allowing to avoid IgRH injection in $39 \%$ of the women who carry D-negative fetuses [11]. In Denmark, a national routine antenatal anti-D prophylaxis program implemented in 2010 guaranteed that administration of IgRH is based on the results of antenatal screening of fetal $R H D$ gene and allows to avoid unnecessary use of prophylaxis in $37.3 \%$ of D-negative pregnant women $[2,12]$. In the Netherlands, since July 1, 2011, both antenatal and postnatal prophylaxis are administered only to women in whom fetal RHD genotyping at gestational week 27 predicts a D-positive fetus [13, 14]. As additional screening methods to guide IgRH prophylaxis, fetal RHD genotyping has also been implemented regionally in France, England, Finland, and Sweden $[15,16]$ and will probably be used for diagnosis by many other countries in the future [17].

Currently, in Argentina, like in other Latin American countries, there are no consensus guidelines for the management of D-negative pregnant women that include non-invasive genotyping of fetal $R H D$. Their availability would make a great impact on regional public health policies both in the management of $\mathrm{D}$-negative pregnant women and with regard to the optimization of the scarce resources available for prophylaxis. In other countries, it is well known that between 20 and $40 \%$ of D-negative pregnant women carry D-negative fetuses [11], and therefore in these cases prophylaxis is not necessary.

Recently, a protocol for non-invasive prenatal $R H D$ genotyping in an Argentinean admixed population has been published [18]. We agree with Clausen [19] when he comments, in reference to the latest research that it is a very good example of how an admixed population was first studied comprehensively and then the results of the population study provided the basis on which an algorithm was designed' ([19] p. 5). However, we also agree with the idea that 'it is possible that the overall algorithm may be simplified to become more suitable for routine analysis when implemented into clinical practice' ([19] p. 5). Herein, we report our experience on the use of a molecular assay for RHD genotyping in the screening of fetal $R H D$ in another very mixed population of pregnant women in Argentina using two exons. We demonstrated a good performance of this simple protocol for clinical application to optimize immunoprophylaxis.

\section{Material and Methods}

This research was designed following the principles established in the Declaration of Helsinki of 1975 and revised in 2013. It was approved by the Training and Teaching Committee of the Fundación Banco Central de Sangre and by the Ethic Committee on Human Research of the Hospital Materno Provincial Dr. Raúl Felipe Lucini in accordance with local and national regulations.

\section{Sample Preparation}

Peripheral venous blood was obtained from D-negative pregnant women. Plasma was separated from the cellular component within $72 \mathrm{~h}$ of sampling. Initially, the blood was centrifuged at $1,600 \times g$ for $10 \mathrm{~min}$, and the maternal plasma fraction was separated from the red blood cells (being careful not to contaminate the sample with maternal cells). The plasma was then centrifuged at $11,000 \times g$ for $10 \mathrm{~min}$ to ensure that all remaining cells were removed. Finally, the supernatant was transferred to $1.5-\mathrm{ml}$ tubes. Plasma samples were stored at $-20{ }^{\circ} \mathrm{C}$ to $-30{ }^{\circ} \mathrm{C}$ until used.

cffDNA Extraction

cffDNA extraction was performed with the QIAamp DNA Blood Mini kit (Qiagen, Hilden, Germany) following the manufacturer's instructions, with some modifications. The initial volume of plasma used was $800 \mu \mathrm{l}$, and the extracted nucleic acid was eluted in $50 \mu$ of elution buffer.

Real-Time Polymerase Chain Reaction

To determine the presence of fetal RHD gene in cffDNA, we performed realtime polymerase chain reaction (PCR) using TaqMan fluorogenic probes and
Blanco/Giacomi/Slobodianiuk/Frutos/Carrizo/ Fanin/Culasso/Gallego 
ABI Prism 7500 Sequence Detector (Applied Biosystems, Foster City, CA, USA) for simultaneous amplification of sequences of exons 5 and 10 of the RHD gene. Amplification and detection of exons 5 and 10 were carried out using primers Exo5-F (5'-CGCCCTCTTCTTGTGGATG-3'), Exo5-R (5'-GAACACGGCATTCTTCCTTTC-3'), Exo10-F (5'-TGCCTGCATTTGTACGTGAGA - 3 ') and Exo10-R ( $5^{\prime}$-AGTGCCTGCGCGAACATT- $\left.3^{\prime}\right)$ to a final concentration of $300 \mathrm{nmol} / \mathrm{l}$ and probes Exo5 (VIC-TCTGGCCAAGTTTCAACTCTGCTCTGCT-TAMRA) and Exo10 (FAM-CATGACAGCAAAGTC-MGB) at a final concentration of $200 \mathrm{nmol} / \mathrm{l}$, respectively. Primers and probe for RHD exon 5 testing are selected according to Finning et al. [20]. Primers and probes for RHD exon 10 as well as for $S R Y$ testing were kindly provided by Nuria Nogues, of the Immunohematology Laboratory, BST, Barcelona, Spain.

To confirm the presence of cffDNA, a sequence of the Homo sapiens sex determining region Y (SRY, GenBank Accession number NC_000024.10, region 2787803...2787865) was amplified and detected using primers SRY-F ( $5^{\prime}$-GAGCAGTCAGGGAGGCAGAT- $\left.{ }^{\prime}\right)$ and SRY-R (5'-GCAAAACATGGTAATTCAGTAACGTT- $3^{\prime}$ ) at a final concentration of $300 \mathrm{nmol} / \mathrm{l}$ and the SRY probe (FAM-AGCAGGGCAAGTAGT-MGB) at $200 \mathrm{nmol} / \mathrm{l}$. The reactions were performed in a final volume of $20 \mu \mathrm{l}$ using Taqman Universal PCR Master Mix (Applied Biosystems). After initial incubation at $50^{\circ} \mathrm{C}$ for $2 \mathrm{~min}$, denaturation at $95^{\circ} \mathrm{C}$ for $10 \mathrm{~min}$ and 452 -step cycles of $95^{\circ} \mathrm{C}$ for $15 \mathrm{~s}$ and $60{ }^{\circ} \mathrm{C}$ for 1 min was performed. Each sample was assayed in duplicate in a uniplex reaction for exon 10 and in triplicate in a multiplex reaction for the exon 5 sequence of the RHD gene and the $\mathrm{Y}$ chromosome sequence. The last sequence was limited to male fetuses as a control to detect cffDNA. This approach is used since 2004 at the Immunohematology Laboratory, Banc de Sang i Teixits, Barcelona, Spain, for prenatal RHD genotyping in Catalonia.

\section{Samples Included in the Study}

For implementation and regional validation of the molecular technique, a 10 -month prospective study was carried out. Samples from $120 \mathrm{D}$-negative pregnant women who attended the Department of Hemotherapy and Immunohematology of the Hospital Materno-Provincial Dr. Raúl Felipe Lucini, Córdoba, Argentina, were obtained. Gestational age at time of blood collection ranged between 16 and 42 weeks. The number of plasma samples of pregnant women necessary and sufficient to validate the sensitivity and specificity of the method was defined based on the average number of $\mathrm{D}$-negative pregnant women who attended the mentioned department in a period of 12 months prior to the beginning of the study, in addition to the frequency of D-negative and D-positive infants born from that group during the same period. The determination of fetal RHD genotype was performed in samples collected during prenatal routine controls. Neonates' phenotype was determined in a blood sample from heel prick, $72 \mathrm{~h}$ after birth.

\section{Interpretation of the Results}

The fetuses were considered D-positive (detection of fetal RHD gene) when the two replicates of exon 10 and at least two of the three triplicates of exon 5 were positive. D-negative fetuses (negative detection of $R H D$ gene) were defined when none of the replicates of exons 5 and 10 amplified. In case of male fetuses, at least two of the triplicates of the SRY exons had to turn out positive to confirm the presence of fetal DNA.

Due to the fact that in the presence of $R H D$ pseudogene, $R H D-C E-D$ hybrid or some D variants (DVI type III or DBT), exon 5 does not amplify [17], in cases of positive amplification of both replicates of exon 10 under absence of amplification of exon 5 the samples should be classified as inconclusive. In such cases, additional studies with other techniques are required to elucidate diagnosis.

When both exons amplified but there was a considerable difference $(\geq 4 \mathrm{cy}$ cles) between the threshold cycles (Ct) of the replicates of exon 10 with respect to exon 5, the fetus was considered D-positive as well. Even though in these cases complementary assays are required to characterize possible RHD variants, the unequivocal diagnosis of D-positive fetus makes this unnecessary in the context of clinical management of D-negative pregnant women. In case of discordance in the analysis of the results (discordance in amplification of duplicates of exon 10 or non-concordance in amplification of the two exons), the re- sult was considered inconclusive, and a new sample was requested. When it was not possible to obtain a new sample, the result was defined as inconclusive and excluded from the final analysis.

\section{Neonatal Phenotype}

The results obtained in maternal plasma were compared to the newborns' phenotype using a blood sample from heel prick obtained within $72 \mathrm{~h}$ after birth. Detection of D antigen in neonates was performed by Grifols DG Gel Newborn (Diagnostic Grifols S.A., Barcelona, Spain). In pregnant women who discontinued routine controls or delivered in a different institution, it was not possible to match maternal results with the offspring phenotype; for this reason these data were not included in the final analysis.

\section{Analytical Evaluation}

The analytical performance of the assay was evaluated by accuracy, sensitivity, specificity, positive predictive value (PPV) and negative predictive value (NPV) according to Agresti [21]. Confidence intervals (CI) were estimated according to Haldane [22] and Anscombe [23] correction.

\section{Results}

Real-time PCR was implemented and applied in plasma samples of 120 pregnant women for fetal RHD genotyping and detection of $S R Y$ sequence. Nine of the 120 samples studied presented non-conclusive results and were excluded from final analysis. Among these, $4 / 120(3.33 \%)$ corresponded to women who did not give birth at the Hospital Materno Provincial; therefore, the phenotype of these newborns could not be determined. In another 4/120 (3.33\%) cases, samples had pre-analytical problems, and it was not possible to obtain new samples before delivery. The remaining inconclusive case, $1 / 120(0.8 \%)$, presented repeated reactive results for the amplification of exon 10 (Ct 35) but negative detection of exon 5. It was not possible to obtain a new sample of this patient because she did not attend to subsequent medical consultations neither before nor after delivery.

In the remaining 111 samples, the concordance between the obtained genotype and the fetal phenotype could be compared and analyzed. The prediction of fetal phenotype, both for RHD factor and fetal sex was accurate in all samples, showing 87 (78\%) RHDpositive neonates and 24 (22\%) RHD-negative ones; 57 (51\%) were males and 54 (49\%) females.

The analytical performance of the assay to determine RHD status was as follows: sensitivity $100 \%$ (95\% CI $98.85-100 \%)$, specificity $100 \%$ (95\% CI $95.83-100 \%)$, and accuracy $100 \%$. The PPV for RHD status was $100 \%$ (95\% CI 98.85-100\%). The accuracy of the assay for detection of $S R Y$ sequence was $100 \%$, sensitivity and specificity was $100 \%$ (95\% CI $98.148-100 \%)$ and 100\% (95\% CI 98.246-100\%), respectively. The PPV to detect the SRY sequence of Y chromosome in male fetuses was 100\% (95\% CI 98.148-100\%). $53 \%$ of the $120 \mathrm{D}$-negative pregnant women included in the study were in the third trimester of pregnancy while $47 \%$ were in the second trimester. All pregnant women were born in Argentina and had a multiethnic descent profile: Asian 2.5\%, European 34\%, Argentine $42.5 \%$, Native population $8 \%$ and unknown $13 \%$.

Of the total number of pregnant women, 36 (30\%) were pregnant for the first time, and 84 (70\%) had been pregnant before. In 
the group with previous pregnancies, 22 (26\%) had had only 1 previous pregnancy, 28 (33.2\%) 2 gestations, 14 (17\%) 3, 10 (12\%) 4, 6 (7\%) $5,1(1.2 \%) 6,1(1.2 \%) 9$, and 2 (2.4\%) 10 gestations. 35 (42\%) had previous history of spontaneous abortions; 24 (69\%) of them reported one previous abortion, 7 (20\%) two, and 4 (11\%) three miscarriages, while 8 pregnant women (9.5\%) referred history of a newborn died at birth and $5(6 \%)$ had had a newborn with different clinical symptoms (from cholestasis to mental retardation and heart failure). Among all $\mathrm{D}$-negative pregnant women carrying RHD-negative fetuses, 10 were pregnant for the first time and 14 reported history of previous pregnancies.

Among the 111 pregnant women with the defined fetal RHD genotype, 2 showed a significant difference between $\mathrm{Ct}$ amplification in exon 10 (Ct 32) and exon 5 (Ct 41). In these two cases, the fetuses were considered D-positive. Even though complementary studies would be necessary to assess the possibility that a gene is not being expressed in the mothers, the unequivocal diagnosis of fetuses as D-positive is enough to guide prenatal prophylaxis. Thus, complementary assays for RHD variants were not necessary since it exceeded the objective of this study.

\section{Discussion}

We demonstrated that noninvasive fetal $R h D$ genotyping using two sequences of the gene can be enough for clinical application in an admixed population. Thereby, a simple and cost-effective prenatal diagnostic technique can be used to detect and restrict IgRH use to women carrying D-positive children. Fetal $R h D$ testing can also improve the use of postnatal IgRH.

In this study, a simple protocol was applied using exons 5 and 10 , and the results showed that this robust technique of simple implementation allows to determine fetal RHD in maternal blood with high sensitivity, specificity, accuracy, and precision. This technique follows the consensus recommendation to include exon 5 $[12,17,20,24]$, which, in combination with exon 10 , helps to identify RHD-positive / D-positive subjects and to distinguish of RHDpositive / D-negative cases, mostly due to presence of the $R H D$ pseudogen $(R H D \psi)$ or cases of hybrid $R H D-C E-D^{s}$. Thus, this combination avoids false-positive results and also identifies some RHD variants [15].

For the purposes of clinical management of D-negative pregnant women and decision-making regarding prophylaxis, fetal genotyping should be able to predict fetal phenotype with high accuracy. This study demonstrates that in a multiethnic population the use of exons 5 and 10 allows to appropriately differentiate $R H D$-positive / D-positive individuals from $R H D$-positive / D-negative ones. Thus, management of $\mathrm{D}$-negative pregnancies and target antenatal prophylaxis could by successfully achieved.

Even though RHD $\psi$ or RHD-CE- $D^{\mathrm{s}}$ in $R H D$-positive / D-negative individuals could not be differentiated with the study of exons 5 and 10 and required complementary tests, these variants of the RHD genotype have no impact in clinical routine since only the expressed phenotype is relevant to clinical management of these patients.
The usefulness of the analysis of these two exons in a heterogeneous population to identify the fetal phenotype has been corroborated by a recent study of Trucco Boggione et al. [18], in which exons 4 and 7 were also studied. In spite of the inclusion of more than two exons in the suggested algorithm, it was necessary to carry out complementary studies to characterize RHD-positive / Dnegative individuals. In the Argentinean population, approximately $2 \%$ of D-negative individuals harbor an RHD allele $[25,26]$. This information has been recently corroborated in a study by Trucco Boggione et al. [18] and in our results, supporting that the assessment of noninvasive prenatal RHD using exon 5 and 10 is capable to appropriately predict fetal phenotype and guide target antenatal prophylaxis. For this reason, we propose that this technique should be routinely implemented as alternative diagnostic method for prenatal RHD typing in the management of D-negative pregnancies due to its high accuracy and cost-effectiveness, in order to reduce unnecessary administration of antenatal prophylaxis.

In many countries, IgRH is an expensive and limited resource, mainly because it is a biological product obtained from plasma pools of donors with high anti-D titers. According to a report released by the Pan American Health Organization in June 2017 [27], less than half of blood donors are volunteers in Latin America and the Caribbean. The report also reveals that in Latin America only $33.4 \%$ of the volunteers donates blood or blood components at least twice a year, and in Mexico only $2.7 \%$. In these countries, including Argentina, where altruistic repeated donations are the exception and not the rule and where are no public policies for implementation of programs to obtain plasma with high anti-D titers, the raw material for IgRH production is very scarce $[5,6]$. This is the main limitation for IgRH availability, and therefore the administration of prenatal IgRH has been suspended in some cases and in different regions. Thus, in these countries, it is currently necessary to change the cultural habits of reposition and single donation motivated by urgency to a culture based on repeated altruistic donations. In this context, the routine use of fetal $R h D$ genotyping in all D-negative pregnant women as part of an antenatal screening program would be a good measure towards immunoprophylaxis optimization.

On the other hand, it has been recently discussed that routine administration of biological products, such as IgRH, would not be ethically acceptable when molecular methodologies for fetal $R H D$ genotyping are available to identify those women that do not need their administration [10].

Biological products prepared from pools of various donors carry the risk of infection transmission, as it has already been demonstrated in different studies [7-9]. There is a residual risk of viral contamination and a potential risk of prion transmission, which corroborates that IgRH, like any other biological product, should be administered only when it is really necessary [28]. It is necessary to bear in mind that this residual risk also involves the possibility of new emerging viral diseases, as it has occurred during the last 10 years. Emerging pathogens could potentially be transmitted since 
detection techniques or screening programs may not always be available at the time of emergence.

The precautions to consider when using IgRH are various, and, given the current situation, it seems necessary to establish a prophylactic program to optimize the available resources; in terms of fetal-maternal alloimmunization, fetal RHD genotyping allows to identify those cases in which administration of IgRH is really necessary, making the management of $\mathrm{D}$-negative pregnancies more suitable in order to optimize immune prophylactic recourses in clinical practice.

In European countries, $40 \%$ of D-negative pregnant women bear negative fetuses, while in countries like India the frequency is 25\% [17]. Therefore, recognition of fetal RHD status of European and Indian D-negative pregnant women allows to avoid unnecessary administration of IgRH in 40 and $25 \%$ of women carrying Dnegative fetuses, respectively. In our studied population, $22 \%$ of D-negative pregnant women carried D-negative fetuses; therefore, they were not at risk of immunization. These results, in line with what has been recently described by Trucco Bogionne et al [18], corroborate the need of updating antenatal screening programs in regions without consensual guidelines for the use of antenatal prophylaxis.

A simple and cost-effective prenatal diagnostic method for fetal $R H D$ screening should be the base of the prophylactic program for a rational use of IgRH. The high accuracy of fetal genotyping using two sequences of the gene proved that it can be sufficient for clinical application in an admixed population. Thereby, owing to the good performance of this assay, we recommend that prevention policies for admixed populations, like those of America, consider its incorporation for prophylaxis optimization of fetal-maternal alloimmunization. Noninvasive fetal $R h D$ genotyping is highly reliable and can be used to optimize resources targeting antenatal and postnatal IgRH usage.

\section{Acknowledgments}

We are thankful to Dr. Eduardo Muñiz-Díaz and Dra. Nuria Nogués from the Immunohematology Laboratory, Banc de Sang i Teixits, Barcelona, Spain, for kindly transfer the technique; to Dra. Graciela Panzetta Dutari, Researcher of the National Council of Scientific and Technical Research (CONICET) and Associated Professor, Department of Clinical Biochemistry, Facultad de Ciencias Químicas, Universidad Nacional de Córdoba, for her support during the implementation of the technique in our laboratory, and to Dr. Jorge Cámara from the Institute of Virology Dr J.M. Vanella, Facultad de Ciencias Médicas, Universidad Nacional de Córdoba for his technical assistance.

\section{Contribution to Authorship}

SB and SVG conceived and designed the study. SB, SVG, and MCF processed the samples. VSG, LGS, LHC, GEF, and JMC recruited and followed the patients. All authors analyzed the data. SB and SVG designed and wrote the manuscript.

\section{Disclosure Statement}

The authors declare no conflicts of interest relevant to the publication of this article.

\section{References}

1 Liumbruno GM, D'Alessandro A, Rea F, Piccinini V, Catalano L, Calizzani G, Pupella S, Grazzini G: The role of antenatal immunoprophylaxis in the prevention of maternal-foetal anti-RH(D) alloimmunisation. Blood Transfus 2010;8:8-16.

2 Clausen FB, Christiansen M, Steffensen R, Jørgensen S, Nielsen C, Jakobsen MA, Dyhrberg Madsen R, Jensen K, Krog GR, Rieneck K, Sprogøe U, Homburg KM, Grunnet N, Dziegiel MH: Report of the first nationally implemented clinical routine screening for fetal RHD in Dpregnant woman to ascertain the requirement for antenatal RHD prophylaxis. Transfusion 2012;52:752-758

3 Bowman J: Thirty five years of RH prophylaxis. Transfusion 2003;43:1661-1666.

$\checkmark 4$ Parant O: Comparison of the efficacy of different methods for the prevention of anti-D allo-immunization during pregnancy: targeted strategy limited to risk situations or associated with systematic prevention in the 3rd trimester (in French). J Gynecol Obstet Biol Reprod (Paris) 2006;35:1S93-1S103.

5 Torres OW: Marco regulatorio para el control inmunohematológico de la enfermedad hemolítica perinatal en la República Argentina. Rev Hosp Mat Inf Ramón Sardá 2007;26:84-89.

6 Torres OW, Endara AB: Profilaxis de la Enfermedad hemolítica perinatal por Anti-D: consideraciones clínicas y recomendaciones. Revista Argentina de Transfusión 2013;XXXIX:23-30.
7 Kenny-Walsh E: Clinical outcome after hepatitis C infection from contaminated anti-D immunoglobulin. Irish Hepatology Research Group. N Engl J Med 1999; 340:1228-1233.

8 Smith DB, Lawlor E, Power J, O'Riordan J, MsAllister J, Lycett C, Davidson F, Pathirana S, Garson JA, Tedder RS, Yap PL, Simmonds P: A second outbreak of hepatitis $\mathrm{C}$ virus infection for anti-D immunoglobulin in Irland. Vox Sang 1999;76:175-180.

9 Schreir E; Höhne M, Künkel U, Berg T, Hopf U: Hepatitis GBV-C sequences in patients infected with $\mathrm{HCV}$ contaminated anti-D immunoglobulin and among i.v. drugs users in Germany. J Hepatol 1996;25:385-389.

10 Kent J, Farrell AM, Soothilln P: Routine administration of anti-D: the ethical case for offering pregnant woman fetal RHD genotyping and a review of policy and practice. BMC Pregnancy Childbirth 2014;14:87.

11 Minion JM, Gerard CH, Chantraine F and Nisolle M: Anti-D prophylaxis review in the era of foetal RHD genotyping. J Blood Disord Transfus 2015;6:302.

12 Daniels G, van der Schoot CE, Olsson ML: Report on the fourth international workshop on molecular blood group genotyping. Vox Sang 2011;101:327-332.

13 Haas M, Van der Ploeg CP, Scheffer PG, Verlinden DA, Hirschberg H, Abbink F and van der Schoot CE: A nation-wide fetal RHD screening programme for targeted antenatal and postnatal anti-D. ISBT Sci Ser 2012;7:164-167.
14 Hass M, Thurik FF, Van Der Ploeg CP, Veldhuisen B, Hirschberg H, Soussan AA, Woortmeijer H, Abbink F, Page-Christiaens GCML, Scheffer PG, van der Schot $\mathrm{CE}$ : Sensitivity of fetal RhD screening for safe guidance of targeted anti-D immunoglobulin prophylaxis: prospective cohort study of nationwide programme in Netherlands. BMJ 2016;355:15789.

15 Haimila K, Sulin K, Kuosmanen M, Saraneva I, Korhonen A, Natunen S, Tuimala J, Sainio S: Targeted antenatal anti-D prophylaxis program for $\mathrm{RhD}$-negative pregnant women - outcome of the first two years of a national program in Finland. Acta Obstet Gynecol Scand 2017;96:1228-1233.

16 Clausen FB, Damkjaer MB, Dziegiel MH: Noninvasive fetal RHD genotyping. Transfus Apher Sci 2014;50: 154-162.

17 Parchure DS, Kulkarni SS: Noninvasive fetal RHD genotyping from maternal plasma. Glob J Transfus Med 2016;1:21-28.

18 Trucco Boggione C, Luján Brajovich ME, Mattaloni SM, Di Mónaco R, García Borrás SE, Biondi CS, Cotorruelo C: Genotyping approach for non-invasive foetal RHD detection in an admixed population. Blood Transfus 2017;15:66-73.

19 Clausen FB: Non-invasive foetal RhD genotyping in and admixed population. Blood Transfus 2017;15:4-5. 
20 Finning KM, Martin PG, Soothill PW, Avent ND: Prediction of fetal $\mathrm{D}$ status from maternal plasma: introduction of a new noninvasive fetal RHD genotyping service. Transfusion 2002;42:1079-1085.

21 Agresti A: An Introduction to Categorical Data Analysis. New Jersey, Wiley-Interscience, 2007, pp 372.

22 Haldane, J: The measures of variation. Evolution 1955 9:484.

23 Anscombe, FJ: On estimating binomial response relations. Biometrika 1956;43:461-464.
Maddocks DG, Alberry MS, Attilakos G, Madgett TE, Choi K, Soothill PW, Avent ND: The SAFE project: towards non-invasive prenatal diagnosis. Biochem Soc Trans 2009;37:460-465.

25 Trucco Boggione C, Luján Brajovich M, Tarragó M, Mattaloni SM, Biondi CS, Muñiz-Diaz E, Nogués N, Cotorruelo CM: Molecular structures identified in serologically D- samples of an admixed population. Transfusion 2014;54:2456-2462.

26 Luján Brajovich M, Trucco Boggione C, Nogués N, Racca A, Muñiz Diaz E, Cotorruelo C: Alelos RHD nulos en pacientes RHD negativos de una población de Rosario. Rev Arg Transfus 2010;36:125-139.
27 Pan American Health Organization: Supply of blood for transfusion in Latin Amrica and Caribbean Countries, 2014 and 2015. PAHO, Washington DC, 2017.

28 Teitelbaum L, Metcalfe A, Clarke G, Parboosingh JS, Wilson RD, Johnson JM: Clinical outcome after hepatitis $\mathrm{C}$ infection from contaminated anti-D immune globulin. Irish Hepatology Research Group N Engl J Med 1999;340:1228-1233. 